\title{
LA AGENDA OCULTA DEL TRABAJO INVISIBLE: APROXIMACIÓN TEÓRICA Y CATEGORIZACIÓN
}

\section{Inmaculada Hurtado inmaculada.hurtado@uch.ceu.es \\ Universidad CEU-Cardenal Herrera}

Elena Juaristi Besalduch

elena.juaristi@uch.ceu.es

Universidad CEU-Cardenal

Herrera

\author{
Elia Saneleuterio \\ elia.saneleuterio@uv.es \\ Universitat de València
}

María García-Alcober

maria.garcia3@uch.ceu.es

Universidad CEU-Cardenal

Herrera

\section{Rocío López-García-Torres \\ rocio.lopez@uch.ceu.es \\ Universidad CEU-Cardenal \\ Herrera}

Ruth Abril Stoffels

rabril@uch.ceu.es

Universidad CEU-Cardenal

Herrera

Recibido: 24-02-2015

Aceptado: 26-04-2015

\section{Resumen}

Los estudios sobre corresponsabilidad en el trabajo doméstico y en la sostenibilidad de la vida han valorado las desigualdades entre hombre y mujeres en el reparto de las funciones domésticas y de cuidado, pero - hasta el momento - han dejado de lado algunas tareas que por sus características resultan más difusas. Nuestra investigación pretende comprobar cuáles son las tareas que siguen siendo invisibles actualmente, de modo que puedan ser incorporadas para futuros análisis y colaborar, con esta visibilización, a su consideración social y a la corresponsabilidad plena como aspiración imprescindible de igualdad entre sexos.

Palabras clave: Trabajo invisible, cuidados, visibilización, género, uso del tiempo, reparto por sexos, mujer, corresponsabilidad.

\begin{abstract}
Reports and statistics about joint responsibility in household work and in the sustainability of life have assessed the inequalities between men and women in the distribution of care and domestic functions up until the present moment. Our hypothesis is that some general household tasks have been ignored. Our research aims to check which tasks remain invisible even today, so that they can be incorporated into future analysis. The last objective is to collaborate with this visibility towards its social assessment, a first step to true joint responsibility as an indispensable aspiration of gender equality.
\end{abstract}

Keywords: Invisible work, care, visibility, genre, time uses, distribution between sexes, woman, joint responsibility. 


\section{Introducción. Hacia una definición de la agenda oculta}

En España la investigación sobre lo que por entonces se entendía como trabajo invisible se inició a mediados de la década de los 70 (Durán, 1975). Pero no fue hasta los años 90 (Álvaro Page, 1996a) cuando este tipo de estudios alcanzaron una consolidación definitiva, dando lugar a un avance considerable en el conocimiento que por entonces se tenía sobre el trabajo no remunerado. Y, como sabemos, tal y como arrojaban las encuestas en ese momento ${ }^{1}$, la dedicación al trabajo doméstico y cuidado de los miembros de la familia no se repartía de forma aleatoria entre la población, sino que estaba fuertemente asociada a las mujeres, incluso en aquellas con empleo remunerado.

Durante el siglo XX se identificó como trabajo invisible la suma de actividades domésticas y de cuidado familiar, mayormente realizadas por mujeres, que no eran retribuidas ni reconocidas como trabajo. Recordemos que, hasta hace poco, el trabajo del hogar era trabajo invisible: ni remunerado ni reconocido y, por lo tanto, sin valor económico ni social (García Sainz, 2005: 40). De hecho, cuando se le preguntaba a una mujer si trabajaba, se sobreentendía que se estaba haciendo referencia exclusivamente al trabajo remunerado y no al resto de tareas, no identificadas como "trabajo".

Sin embargo, en la medida en que las responsabilidades domésticas se han ido externalizando o mercantilizando, y desde que comenzó a generalizarse la corresponsabilización con el varón (Ballester Pastor, 2012), este trabajo ha empezado a ser apreciado, aunque sin adquirir todavía la visibilidad y valoración necesarias para el logro de la igualdad social en los hogares, a pesar incluso de medidas legislativas explícitas en nuestro país (Martín, León y Guirao, 2013) ${ }^{2}$. Asimismo, cuando se producen situaciones como las

\footnotetext{
${ }^{1}$ Encuesta de actividades (Ramos, 1990), encuestas sobre uso del tiempo (CIRES, 1991; 1993a; 1993b; 1996), encuesta a familias con niños escolarizados (CSIC, 1994), encuesta de actividades no remuneradas (CSIC, 1995).

${ }^{2}$ Consúltese la Ley 39/1999, de 5 de noviembre, para promover la conciliación de la vida familiar y laboral de las personas trabajadoras (España, 2999), que trasladó a la legislación española las directrices marcadas por la normativa internacional y comunitaria, superando los niveles mínimos de protección marcados en las mismas.
} 
derivadas de separaciones conyugales y divorcios, la necesidad de dar una traducción pecuniaria a la aportación que las mujeres han hecho en el seno de las familias ha requerido una estimación económica de las mismas. Así, pues, la pretensión de hacer partícipe al varón en estas tareas al $50 \%$ ha supuesto un aligeramiento para quienes normalmente las realizaban —las mujeres - y un mayor reconocimiento económico de su importancia.

En todo caso, es preciso indicar, como afirman Rodríguez y Larrañaga (2003), que en este proceso de incorporación del varón a las tareas domésticas y familiares persisten desigualdades en cuanto a la asignación por sexo de las distintas tareas. Por ejemplo, en la asignación de tareas domésticas a los hombres se produce una "especialización” que resulta generalmente desfavorable para las mujeres tanto en cantidad como en calidad y que puede analizarse en términos de territorialización (Saneleuterio, 2010): habitualmente, las mujeres suelen ocuparse de las tareas menos discrecionales, más laboriosas y que deben realizarse a diario, como la preparación de los alimentos, la limpieza y el cuidado de la prole, mientras que los hombres se ocupan de tareas más esporádicas y visibles como el bricolaje, las relaciones con la comunidad de vecinos o los trámites burocráticos.

En consecuencia, tras los cambios más aparentes, aún perviven muchas resistencias, dado que el cambio social se produce normalmente de forma lenta y compleja. Por ello, a pesar de que en las últimas décadas nos hemos ido acercando a la paridad entre hombres y mujeres, también es una realidad que existen relaciones desiguales y que no se ha producido un reparto equitativo de este tipo de responsabilidades. Efectivamente, el tiempo que se dedica a estas tareas implica desigualdad, según han demostrado estudios como el de María Luisa Setién

Asimismo, mencionamos la Ley Orgánica 3/2007, de 22 de marzo, para la igualdad efectiva de mujeres y hombres (España, 2007). Dicho texto establece que el principio de igualdad de trato entre mujeres y hombres supone la ausencia de toda discriminación, directa o indirecta, por razón de sexo y, especialmente, las derivadas de la maternidad, la asunción de obligaciones familiares y el estado civil, así como que la igualdad de trato y de oportunidades entre mujeres y hombres es un principio informador del ordenamiento jurídico.

En el ámbito autonómico, la Ley 9/2003, de 2 de abril, para la Igualdad entre Mujeres y Hombres (Generalitat Valenciana, 2003) proclama en su preámbulo la posibilidad de conciliar la actividad profesional con la vida familiar como uno de los pilares básicos dentro del ámbito laboral de actuación. Además, el I Plan de Igualdad entre Mujeres y Hombres de la administración de la Generalitat 20102012 previó numerosas normas al respecto (Conselleria de Justicia y Administraciones Públicas, 2010). $C f r$. el informe correspondiente de la Generalitat Valenciana (2013).

Finalmente, es preciso mencionar el Decreto 133/2007, de 27 de julio, sobre las condiciones y requisitos para el visado de los Planes de Igualdad de las Empresas de la Comunidad Valenciana (Conselleria de Bienestar Social, 2007), como medida que busca promocionar y generalizar la adopción de medidas de conciliación en el tejido empresarial valenciano. 
(2003) o Aguirre, García Sainz y Carrasco (2005), sobre todo si tenemos en cuenta que todavía permanecen en la agenda oculta. María del Rosario Marcos Santiago (2005; 2009), por ejemplo, insiste que ciertos tiempos y ciertos trabajos femeninos, incluso siendo visibles, han necesitado mucho tiempo para alcanzar cierto reconocimiento social y económico ${ }^{3}$. Incluso en estos casos, en los que las llamadas tareas invisibles han sido visibilizadas y se reparten entre los miembros del hogar, las mujeres siguen dedicando más tiempo a las tareas domésticas o familiares $\mathrm{y}$, sobre todo, sintiendo que la responsabilidad principal recae sobre ellas - a cambio, muchas veces, de no caer en situaciones de marginalidad femenina (Cárdenas y Undurraga, 2014: 287).

De ahí que hablemos del trabajo que continúa estando oculto en la actualidad. Se trata de aquellas tareas y responsabilidades que, vinculadas a la familia o al hogar, nunca aparecen en las estadísticas de uso del tiempo o en los cómputos de asignación del trabajo doméstico (Durán, 1997), pero que, de hecho, constituyen labores esenciales para el sostenimiento del mismo y que suponen desigualdades por razón de sexo. Partimos, pues, de la premisa de que existe una agenda oculta del trabajo invisible $\mathrm{y}$, además, de que esta es asumida fundamentalmente también por las mujeres, convirtiéndose en un foco de desigualdad.

Así pues, con el concepto de agenda oculta hemos tratado de indagar en aquellas tareas que todavía están marginadas en la agenda del trabajo doméstico y de cuidado de la familia. Aquellas que incluso son obviadas por las mismas personas que las desempeñan cuando son interpeladas a describir la relación de trabajos que realizan en el ámbito doméstico y familiar a lo largo de sus jornadas. Aun pasando desapercibidas a nivel de nombrarlas o cuantificarlas conscientemente, todas ellas, sumadas, suponen una dedicación importante en términos de asunción de responsabilidades y de tiempo empleado para desempeñarlas, aparte de un estrés añadido a quienes normalmente las realizan. Además, estas tareas no son reconocidas socialmente, porque muchas de ellas no son consideradas ni siquiera como "productivas".

El problema residiría en que, como afirma Royo (2003), en algunas personas existen sesgos de género sobre el trabajo doméstico que desde un nivel inconsciente o preconsciente orientan la conducta. Así pues, tratamos de ampliar y desagregar el trabajo doméstico y de

\footnotetext{
${ }^{3}$ Los estudios sociohistóricos de género insisten en la pervivencia y asunción secular de esta falta de reconocimiento. Las referencias al oikos nos devuelven, por ejemplo, una imagen de la mujer recluida en el gineceo; las tareas femeninas en la casa griega eran "cuidar de los hijos, de los bebés, hasta que cumplían siete años, cuidaban a los enfermos, asistían a los muertos, tejían e hilaban y administraban la casa y a los trabajadores domésticos" (Fernández García, 2009: 15).
} 
sostenibilidad familiar con el fin de obtener herramientas más precisas de análisis de la cantidad, variedad y cualidad de las tareas de esta agenda más invisibilizada. De este modo podremos valorar la carga de su asunción tanto desde su vertiente temporal como psicológica.

La finalidad de este estudio es concretar la agenda oculta del trabajo invisible en el ámbito familiar y doméstico en la actualidad, así como identificar las tareas que la integran. Se trata de una primera aproximación exploratoria y descriptiva, así como una sistematización de aquellas tareas domésticas, de cuidado y atención que no han quedado reflejadas en los estudios que sobre esta materia se han realizado.

A partir de su reconocimiento y clasificación, hemos tratado de identificar aquellas tareas que en este proceso de visibilización no han sido todavía sacadas a la luz ${ }^{4}$. Los estudios que consideran categorías amplias - limpieza, cuidado, compra, cocina- parecen incluirlas, pero cuando comprobamos las actividades concretas en las que se despliegan, observamos que no suelen venir reflejadas. Por ejemplo, en el caso de la compra se incluyen ir al supermercado, cargar los productos y pagarlos. Sin embargo, no queda claro si también se está considerando la detección de las necesidades de consumo y la elaboración de la lista de la compra, tareas que también implican un esfuerzo temporal y psicológico en quien las realiza. Así se obvian muchas tareas más difícilmente identificables — porque no implican un esfuerzo físico- o cuantificables en términos de tiempo — dado que, por sus características, son simultaneables—. Estas circunstancias han llevado al hecho de que no sean valoradas pese a que suponen una carga para quien las asume.

La constatación de estas tareas llevada a cabo en este estudio pretende, pues, abrir nuevas vías de investigación en este campo y hacer perceptible una realidad que, por invisible, no es valorada, ni por los que la realizan ni por los que se benefician de ella: el resto de miembros de la unidad familiar en la que se realiza y la sociedad en general. Como horizonte, esta investigación también pretende "el conocimiento de la estructura e infraestructura de los fenómenos sociales, que permita explicar su funcionamiento con el propósito de poder llegar a su control, reforma y transformación” (Sierra, 2003: 33).

\footnotetext{
${ }^{4}$ Más allá de aquello que sigue llamándose trabajo invisible pero que, como consecuencia del desarrollo social y los informes y estudios realizados, ya no lo es tanto.
} 


\section{Metodología y diseño del estudio}

En los últimos tiempos se ha ido configurando de forma progresiva en las Ciencias Sociales y Humanas un enfoque de investigación asociado a perspectivas de corte interpretativo y también sociocrítico. Es el caso de nuestra investigación que, teniendo en cuenta el objeto de estudio, nos permite definir nuestro trabajo como un estudio analítico, descriptivo y desde la reflexión crítica.

El estudio se ha realizado en distintas fases: una primera de análisis bibliográfico y de documentación existente, para determinar el estado de la cuestión y poder identificar lo que ha quedado al margen en los estudios del denominado habitualmente como trabajo invisible. En la segunda fase, desde un enfoque cualitativo, utilizamos la técnica focus groups o grupos de discusión ${ }^{5}$ con el fin de obtener información sobre las tareas que tanto hombres como mujeres consideran que todavía permanecen invisibilizadas, profundizar en el discurso de género de la división del trabajo y en sus fisuras.

Para escoger la muestra de los grupos de discusión tomamos como referencia a mujeres y hombres que cumplieran el perfil de persona con responsabilidades familiares, que convivieran con hijos en edad escolar o tuvieran mayores a su cargo.

Frente a otro tipo de entrevistas grupales, la modalidad seleccionada presenta ventajas tales como: la discusión grupal provocada por el propio interés que el tema suscita, énfasis más las respuestas que las preguntas, indagación sobre creencias arraigadas, obtención de información no prevista, etc.

Estas son las características fundamentales de los grupos de discusión que formaron parte de nuestro estudio:

- La muestra la componían grupos de 8-10 personas, de padres y madres de alumnos

en Educación Primaria, casados o en pareja, residentes en la provincia de Valencia. Para

\footnotetext{
${ }^{5}$ Con su origen en la entrevista focalizada (Merton, Fiske y Kendall, 1956), un grupo de discusión hoy en día puede definirse como un grupo reducido, de aproximadamente 8-10 integrantes, que trata durante una o dos horas de algunas cuestiones relativas al tema objeto de investigación de manera libre e informal, guiados por un moderador que dispone de un guion semiestructurado (Núñez y Loscertales, 2003). Se siguen unas normas mínimas de actuación, que son las propias de un grupo participativo (clima adecuado, concreción de objetivos, planificación, diálogo). Los participantes, de un modo hasta cierto punto informal, son conducidos por un coordinador que deberá presentar el tema y guiar las preguntas, siempre evitando el sesgo en el planteamiento. Este moderador al final intentará ofrecer un resumen de lo tratado y llegar a unas conclusiones. Cfr. Morgan (1988) y Krueger (1997).
} 
poder acceder a este perfil de miembros de los grupos y favorecer la amplia cobertura socio-demográfica, se accedió a los candidatos a través de las AMPAS de centros escolares de diferente titularidad: públicos, concertados y privados de la provincia de Valencia.

- Los participantes fueron seleccionados teniendo en cuenta que habían de tener los rasgos citados, y valorando como preferible que tuvieran entre ellos poca relación. La técnica de selección se basó en la invitación telefónica, apoyada por carteles anunciadores y combinada con el procedimiento conocido como "bola de nieve" 6 .

- Para asegurar fiabilidad, llevamos a cabo 6 grupos de discusión, 3 de hombres y 3 de mujeres, por separado, con la intención de evitar sesgos u omisiones en los discursos. Se procuró que la muestra fuera variada en cuanto a edades y modelos familiares para garantizar la diversidad de situaciones y riqueza de las respuestas.

- La moderadora - de acuerdo con las pautas clásicas de los focus groups (Morgan, 1988; Krueger, 1997) - disponía de un guion semiestructrado, diseñado a la luz de aportaciones procedentes de los miembros del grupo de investigación en base a experiencias familiares personales, directas e indirectas, así como, sobre todo, la confrontación de informes y estadísticas.

- Los participantes fueron informados de que la sesión iba a grabarse, de que participaban libremente y de que podían abandonar la sala en cualquier momento. Asimismo, se les aseguró que se tendría en cuenta el compromiso de confidencialidad.

- La duración de los grupos de discusión fue de 1 hora y 30 minutos, aproximadamente.

En una tercera fase se procedió a realizar el análisis de la información recogida en los grupos de discusión. Para ello, se identificaron unidades de significado que, complementadas con algunas propuestas de la bibliografía precedente, sirvieron para elaborar un listado de tareas, buscando la sistematización de este tipo de actividades, y agrupándolas en categorías explicativas según las características compartidas.

\footnotetext{
${ }^{6}$ El incentivo para asegurar —o aumentar, según los casos - la participación consistió en ofrecer merienda y servicio de guardería, así como el anuncio de obsequios para los participantes.
} 


\section{Categorías operativas para el análisis de la agenda oculta del trabajo invisible}

Entendemos por categorización una operación de clasificación de elementos constitutivos de un conjunto por diferenciación, tras la agrupación por género de actividad, a partir de criterios previamente definidos. $\mathrm{Su}$ principal objetivo es suministrar, por condensación, una representación simplificada de datos brutos, es decir, el paso de los datos brutos a datos organizados. En este sentido, los ámbitos de actividad derivados de las tareas manifestadas en los grupos de discusión se podían organizar en las siguientes categorías: cuidados personales; trabajo doméstico; y sociabilidad, descanso y ocio. Se procuró que estas dimensiones no dejaran al margen ninguna de las tareas formuladas.

Hemos utilizado el término cuidados personales para referirnos aquellas tareas de acompañamiento y asistencia que se desempeñan no solo en el ámbito doméstico-familiar, sino preferentemente para el ámbito doméstico-familiar. Esto es, las actividades se desempeñan vinculadas a los lazos y responsabilidades que se tienen en el núcleo familiar y en el hogar, pero no realizándose necesariamente dentro del mismo (Hurtado, 2010). La razón principal es que estas se pueden llevar a cabo, por ejemplo, en el hogar, al aire libre, en un centro público o en una institución privada.

En trabajo doméstico concentramos las tareas que implican un trabajo sobre, para y en el espacio doméstico o el entorno en el que se desenvuelve la familia. Esta dimensión englobaría distintas acciones encaminadas a la limpieza, la organización, la seguridad, la preparación de alimentos, burocracia o trabajos manuales.

Por sociabilidad, descanso y ocio consideramos, en referencia al primer término, todas aquellas acciones encaminadas al mantenimiento de las redes sociales y de afectos, sean familiares, de amistades, vecinales, etc., así como la gestión de todo lo que ello implica. La segunda y tercera, incluirían, además, aquellas tareas que tienen como fin procurar el reposo, el recreo y el esparcimiento familiar.

Una vez agrupadas en su ámbito de actividad, desarrollamos distintas categorías operativas que nos permitieran clasificar las tareas según sus cualidades e implicaciones. Esta categorización tiene una pretensión clasificatoria, pero sobre todo analítica y expresiva. De este modo, elaboramos herramientas conceptuales que nos permitieran discriminar qué elementos están colaborando en invisibilizar estas tareas, así como evaluar el tipo de carga que conllevan 
para quienes las asumen. Pues bien, las categorías implementadas para clasificar las tareas resultaron en 6 dicotomías, que son las que se muestran en la tabla:

\section{Tabla 1. Categorías para la clasificación de las tareas}

\begin{tabular}{|l|}
\hline Previsible/Contingente \\
\hline Física/Mental \\
\hline Simultaneable/No simultaneable \\
\hline Impostergable/Flexible \\
\hline Externalizable/No externalizable \\
\hline Con desplazamiento/Sin desplazamiento \\
\hline
\end{tabular}

Fuente: elaboración propia

Con el binomio Previsible/Contingente, tratamos de discriminar las tareas que pueden ser, por su propia naturaleza, previstas con anterioridad o imprevisibles dada su contingencia. Las tareas contingentes o de reacción son aquellas mediante las que se intenta hacer frente a un suceso inesperado que requiere solución — un niño o niña enferma, se rompe el coche, visita de un familiar, etc.- Este tipo de tareas generan mucho estrés y en su gestión es necesaria capacidad de reacción y de organización. Son aquellas tareas no previstas, ni rutinarias ni cíclicas, que van surgiendo en el acontecer diario y que requieren ser atendidas sin dilación También sería contingente o extrarrutinaria aquella que no responde a algo inesperado, pero que surge con carácter espontáneo y que podemos decidir hacer o no hacer. La asunción de la responsabilidad en las tareas contingentes supone, en quien las asume, mayor estrés, una mayor disponibilidad de tiempo y una jerarquización de las tareas a atender.

Con los términos Cotidiana/Periódica/Ocasional pretendemos distinguir, dentro de las actividades previsibles, las actividades que tienen una secuencia, sea cotidiana o periódica, de las que se realizan de manera puntual pero no son contingencias/reacciones. Las actividades cotidianas son las que el sujeto asume con normalidad por realizarse con carácter diario. En las actividades periódicas, entrarían aquellas que siguen un calendario previsto y cíclico, y para ellas están previstos un tiempo y unos recursos semanal o incluso mensualmente. Son más visibles que las extrarrutinarias, por este carácter repetitivo. Sin embargo, a medida que se espacia la rutina, pierden visibilidad y su organización se hace sobre el calendario de las rutinarias. Por último, las actividades ocasionales serían aquellas que se realizan fuera de la 
rutina diaria, semanal, mensual o incluso anual. Tienen un carácter puntual y pueden ser previsibles o no previsibles.

El binomio Mental/Físico distingue las tareas mentales de gestión, pre-ocupación o previsión de aquellas que implican directamente una acción corporal, la actuación, la intervención, la resolución. Estas tareas llevan a su vez no solo una carga o desgaste distinto, sino que a nivel material y simbólico implican invisibilidad o visibilidad, respectivamente. Las tareas más mentales suelen ser aquellas menos visibles, y por ello también menos reconocidas. Hay muchas actividades que, vistas globalmente, podrían parecer tanto físicas como mentales, por ejemplo "hacer la compra", pero que, en realidad, se pueden separar en acciones distintas llevadas a cabo por personas distintas. En igual sentido, hay actividades que suponen a la vez una actividad física y mental, tal y como la hemos descrito, por ejemplo el cambio de ropa de verano e invierno, sin que se puedan separar en la acción una fase física y otra mental, sino que son simultáneas y llevadas a cabo, en general, por una sola persona. Por otro lado, nosotras hemos clasificado como mental toda aquella actividad que, aunque tenga que reflejarse a través de un acto físico - como hacer una lista o seleccionar un colegio-, lo cierto es que este acto físico no es más que el soporte o exteriorización del mental. Por el contrario, será física toda actividad que suponga algún tipo de movimiento o acción en los que la acción misma sea clave.

En el par Simultaneable/No simultaneable, consideramos tareas simultaneables aquellas que se pueden realizan mientras se realiza otra. La categoría simultaneable en realidad es cuestión de grado: hay tareas más y menos simultaneables. Por ejemplo, preparar la comida podría simultanearse con una llamada de teléfono o con pensar la lista de la compra, pero otras, como llevar al coche a la ITV o realizar la declaración de la renta, no lo son tanto. En todo caso, en estas tareas, siempre se suele considerar una como principal y la otra como secundaria, siendo, en general, aquella que supone un esfuerzo mental, donde media la previsión y el cálculo, la secundaria. Nuestro estudio considera que la mayor asunción de tareas que pueden ser simultaneables implica una situación personal de multitarea $\mathrm{y}$, con ello, un mayor grado de carga.

Con la distinción entre Impostergable/Flexible ponemos el acento en la posibilidad de organización del tiempo disponible en base a la asunción de tareas. Es decir, nos fijamos en si estas deben realizarse en un tiempo concreto y específico, debiendo organizar la agenda en función de ellas, o si, por el contrario, se puede escoger la hora, el día o incluso el mes y, por 
tanto, se incorporan a la agenda buscando un hueco o acomodándolo a otras necesidades. A mayor flexibilidad de las tareas, mayor posibilidad de control del tiempo y de agencia.

En la categoría Externalizable/No externalizable consideramos que la primera permite, en general, confiar en actores externos la realización de una tarea que una unidad económica - empresa o actor público- no puede realizar óptimamente o no quiere ocuparse de ello, frente a este tercer actor que lo puede hacer de una forma más efectiva y eficiente. En nuestra categorización, la externalización supone la posibilidad de confiar en terceros actores la realización de una tarea que es necesaria para el buen funcionamiento de la familia y del hogar o del disfrute del ocio y las relaciones sociales. En nuestro caso es fundamental distinguir entre aquellas labores que se pueden externalizar con familiares cercanos (padres/madres o hermanas/os) sin que haya — ni por el momento pueda haber- un sector privado que cubra estas necesidades, de aquellas disponibles en el mercado. Esto es, podemos encontrarnos determinadas actividades que se pueden externalizar a estas personas cercanas, por la necesidad de conocimientos especiales, sensibilidad especial u otras cuestiones, que pueden explicar que no haya surgido todavía un sector privado capaz de gestionarlo. Por el contrario, tanto en la familia como, especialmente, en el hogar, hay una serie de actividades que se pueden, si se quiere y puede, externalizar a terceras personas o entidades para que lo realicen. Aparte de la sensibilidad especial requerida o no, es importante tener en cuenta que la externalización pagando a un tercero para que desarrolle una tarea depende mucho de los ingresos de la familia. Así, ya en el estudio que llevó a cabo Mariano Álvaro Page (1996b: 291-326) se apreciaba que el incremento en los ingresos familiares determina el menor tiempo dedicado al trabajo doméstico tanto por el varón como por la mujer. Asimismo, esta capacidad económica puede determinar qué actividades que habitualmente no se externalizan podrían llegar a externalizarse, como por ejemplo, la compra de regalos. Del mismo modo, es destacable que la mercantilización de un servicio viene también influida por factores culturales. Un caso llamativo de estos últimos años es la aparición de empresas antipediculosis, es decir, que se dedican a solucionar infestaciones de piojos, tarea que tradicionalmente han asumido madres, abuelas, hermanas y tías, pero en la que parece haber un negocio incipiente. Dependiendo de los contextos culturales, algunas actividades no son en modo alguno externalizables, mientras que en otros, la externalización de esa tarea está más normalizada.

Por último, Con desplazamiento/Sin desplazamiento diferencia entre las tareas que son o implican trayectos y las que no, considerando con ello el consiguiente uso de tiempo y de 
recursos para acometerlas. Esta categoría resulta elocuente al observar cómo se distribuye la movilidad en relación al tipo de actividades. Si bien hombres y mujeres acostumbran a realizar un número similar de desplazamientos, los de las mujeres suelen ser más cortos, y gran parte de ellos están relacionados con tareas asociadas al cuidado (Sánchez, 2012; Carrasco, 2013; Benito, 2014).

Hemos clasificado las tareas formuladas en los grupos de discusión según estas categorías teniendo en cuenta que, por su carácter multifacético y complejo, admiten ser clasificadas desde diversos puntos de referencia. La decisión de ubicar una determinada tarea en una categoría u otra ha venido justificada por las definiciones elaboradas. La mayoría de categorías se encuentran interrelacionadas, por lo que sucede que hay tareas que tienen más de un valor en una misma categoría. La nuestra, si bien no deja de ser una propuesta siempre abierta al debate, es una tentativa para establecer un marco de sentido que visibilice la pluralidad de tareas y de vertientes que condensan, es decir, su cualidad, dónde acontecen, qué implican.

\section{Exposición de resultados}

Los resultados de las clasificaciones y categorizaciones realizadas resultan muy reveladores. En primer lugar analizaremos las 85 tareas encontradas según la dimensión a la que pertenecen para, seguidamente, clasificarlas según sus categorías. Como se puede apreciar en el gráfico 1, que tiene en cuenta la dimensión de las tareas, la mayor parte de tareas corresponden a trabajo doméstico (42\%), seguido muy de cerca por los cuidados personales (38 \%) y siendo la dimensión de "sociabilidad, descanso y ocio" la menos representativa, con un $20 \%$. Esto puede deberse a que las actividades de preparación y gestión de tareas del ámbito del ocio y el descanso no son consideradas como tales tareas, sino como parte del disfrute de las mismas - hacer la paella o elaborar en grupo la lista de la compra, por ejemplo - . Por otro lado, también es cierto que el tiempo mismo dedicado al ocio, descanso y sociabilidad por una persona adulta, en este caso con responsabilidades familiares, es muy limitado. 


\section{Gráfico 1. Clasificación de las tareas según sus dimensiones}

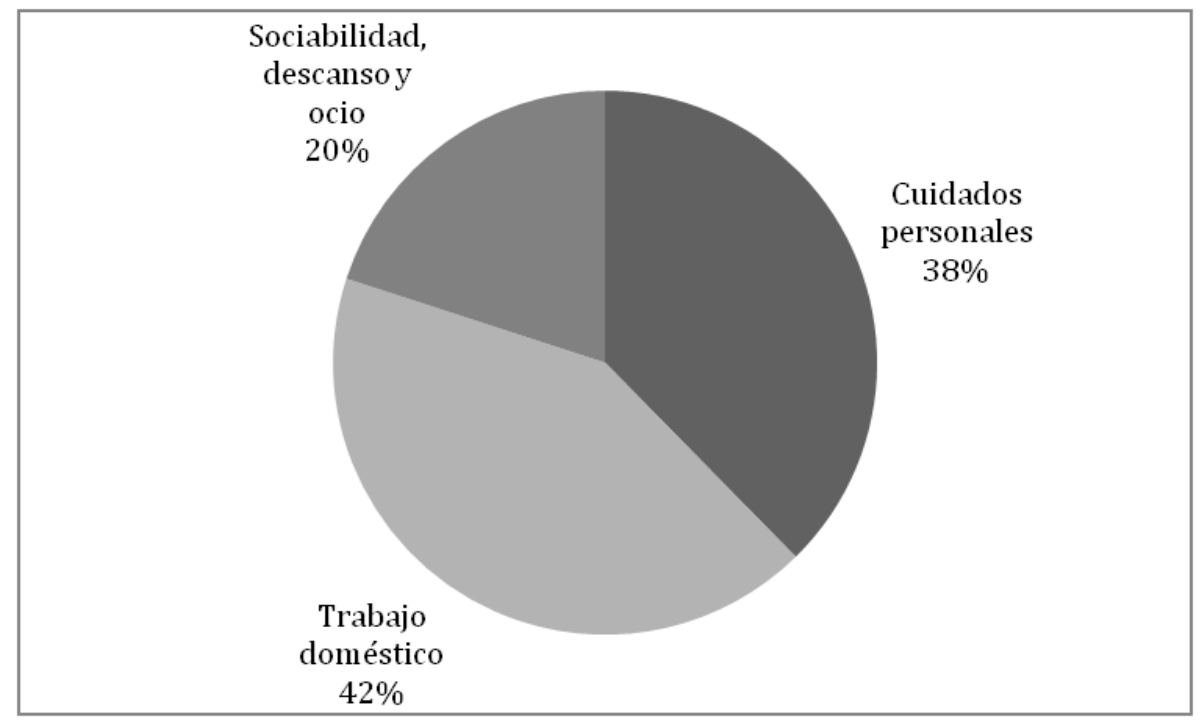

Fuente: elaboración propia

A continuación pasamos a analizar la clasificación de los resultados según sus categorías. En primer lugar, dividimos las tareas entre previsibles y contingentes. Como podemos ver en el gráfico 2, del total de las tareas analizadas, el $74 \%$ de las mismas tiene un carácter previsible, mientras que el $26 \%$ restante tienen carácter contingente. A su vez, las actividades previsibles las hemos clasificado en cotidianas, periódicas y ocasionales. Como podemos apreciar en el mismo gráfico, del total de tareas analizadas solo el $18 \%$ son previsibles cotidianas, quedando un $27 \%$ como previsibles ocasionales y un $29 \%$ como previsibles periódicas.

El reducido porcentaje de las actividades calificadas como contingentes se debe a que realmente el número real de las mismas es menor; sin embargo, hay que considerar que ellas poseen una menor visibilidad tanto social como personal. Muchas veces no somos conscientes del amplio abanico de tareas contingentes que desarrollamos y resolvemos a lo largo del día debido fundamentalmente a su carácter esporádico y a su amplísima diversidad. Ningún estudio debería subestimar este $26 \%$ de actividades contingentes dado que estas actividades realmente suponen un gran estrés, exigiendo gran capacidad organizativa para el que tiene que asumirlas. En una agenda ya organizada hay que incorporarlas necesariamente, ideando 
soluciones creativas en cuestiones no solo de tiempo, sino a veces también de espacio, de búsqueda de personal y de movimiento de agendas de terceros. Por otro lado, parece lógico que las actividades cotidianas previsibles sean menores dentro de nuestro estudio dado que, justamente su cotidianidad permite una mayor visibilidad y por ello difícilmente formarán parte de la agenda oculta.

\section{Gráfico 2. Previsible/Contingente}

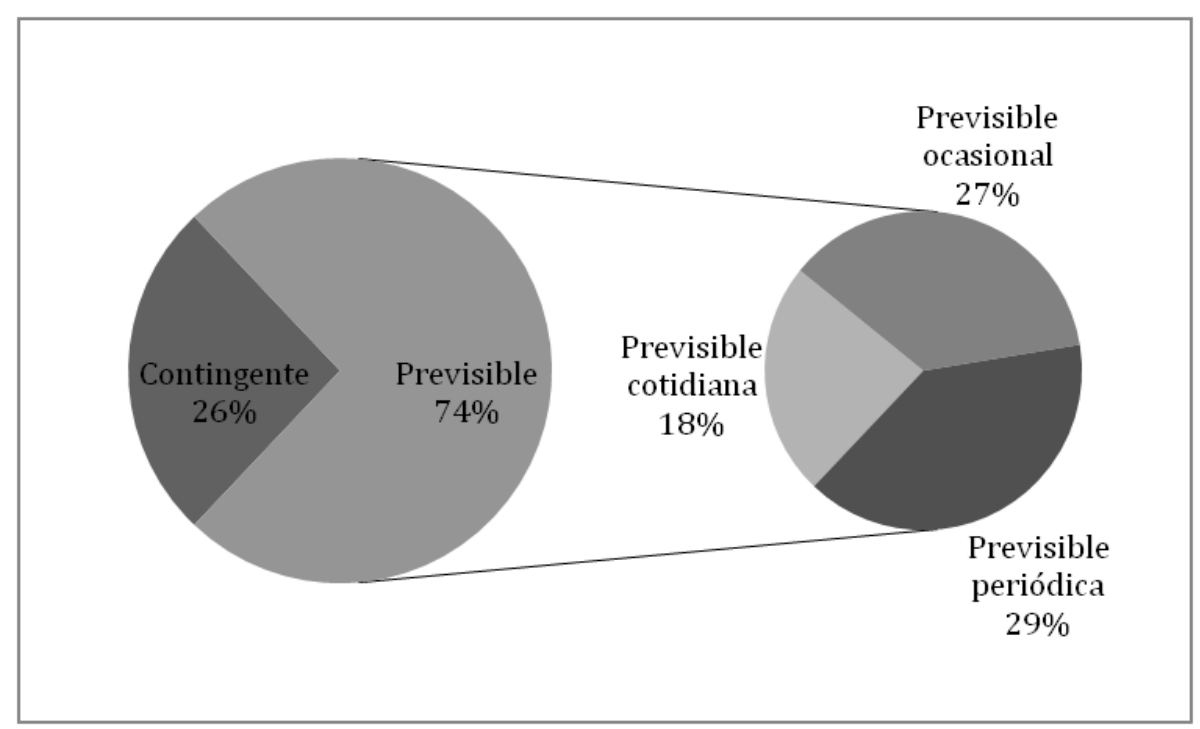

Fuente: elaboración propia

En segundo lugar, hemos diferenciado entre actividades físicas y mentales, teniendo en cuenta que hay actividades que pueden considerarse simultáneamente en ambas categorías. Los resultados pueden apreciarse en el gráfico 3: si consideramos una división tripartita, aparentemente encontramos un reparto equilibrado, tendente a lo físico. No obstante, su porcentaje de $39 \%$ quedaría empequeñecido en una dicotomía que distinguiera simplemente entre implicación mental/no mental. Lo más significativo a nuestros efectos es que las actividades de tipo mental, de organización, planeamiento, cálculo, por sus propias características son bastante ocultas salvo cuando se reflejan luego en una actividad física; en este caso, vemos que el $61 \%$ de las actividades tienen un componente mental que permanece fácilmente invisible. 


\section{Gráfico 3. Física/Mental}

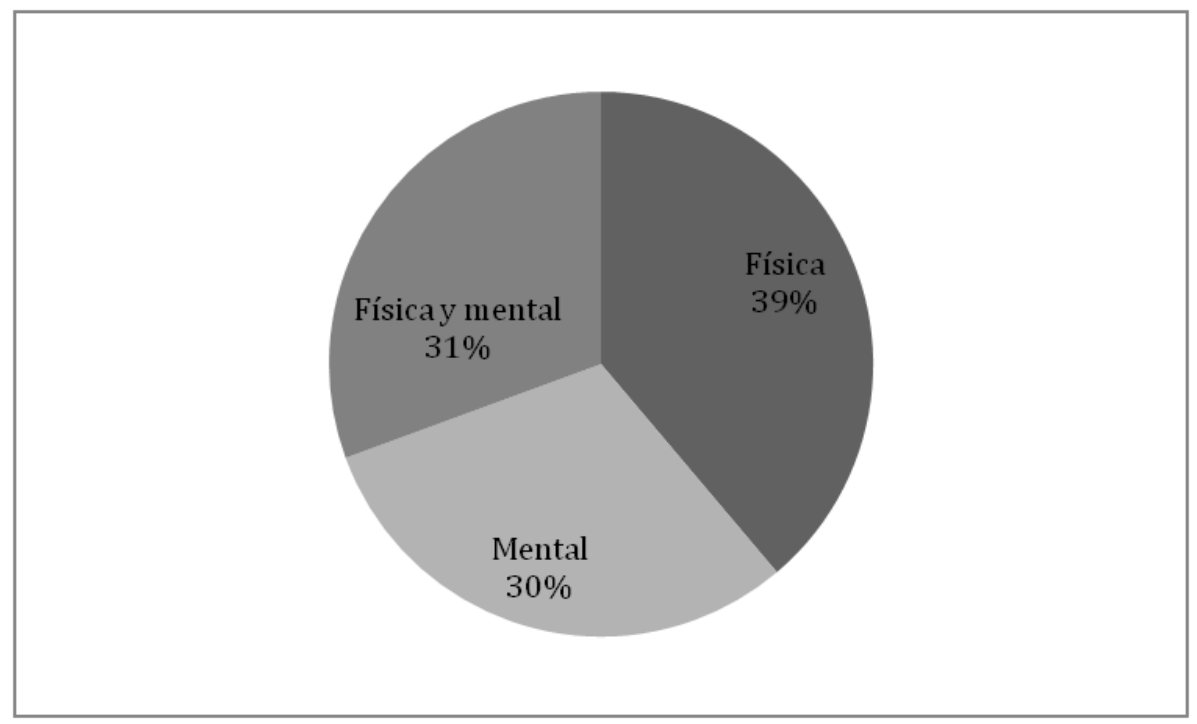

Fuente: elaboración propia

En tercer lugar, analizamos la simultaneidad de las tareas. Como podemos ver en el gráfico 4, el $33 \%$ de las actividades analizadas han sido calificadas como simultaneables, mientras el $67 \%$ restante como no simultaneables. Las expertas ya han señalado, como rasgo del trabajo no remunerado, la multiplicidad y superposición: implican tareas "múltiples y con frecuencia superpuestas" (Durán, 2003: 242). La invisibilidad de las tareas se incrementa cuando esta simultaneabilidad coincide con el carácter mental de las mismas. En referencia a este aspecto, en el gráfico 5 podemos observar que, de las tareas simultaneables, más de dos tercios son mentales, porcentaje que superaría el $90 \%$ si nos fijamos en la dicotomía señalada como implicación mental/no mental. El sensible porcentaje de actividades simultaneables contribuye al fenómeno de la invisibilización de determinadas realidades. De hecho, ante la petición de elaborar un calendario exhaustivo de todo lo realizado en un periodo de tiempo, cuando existen actividades mentales simultaneadas con actividades físicas son las segundas las que suelen anotarse, olvidándose u obviándose las primeras en el registro por parte del sujeto. 


\section{Gráfico 4. Simultaneable/No simultaneable}

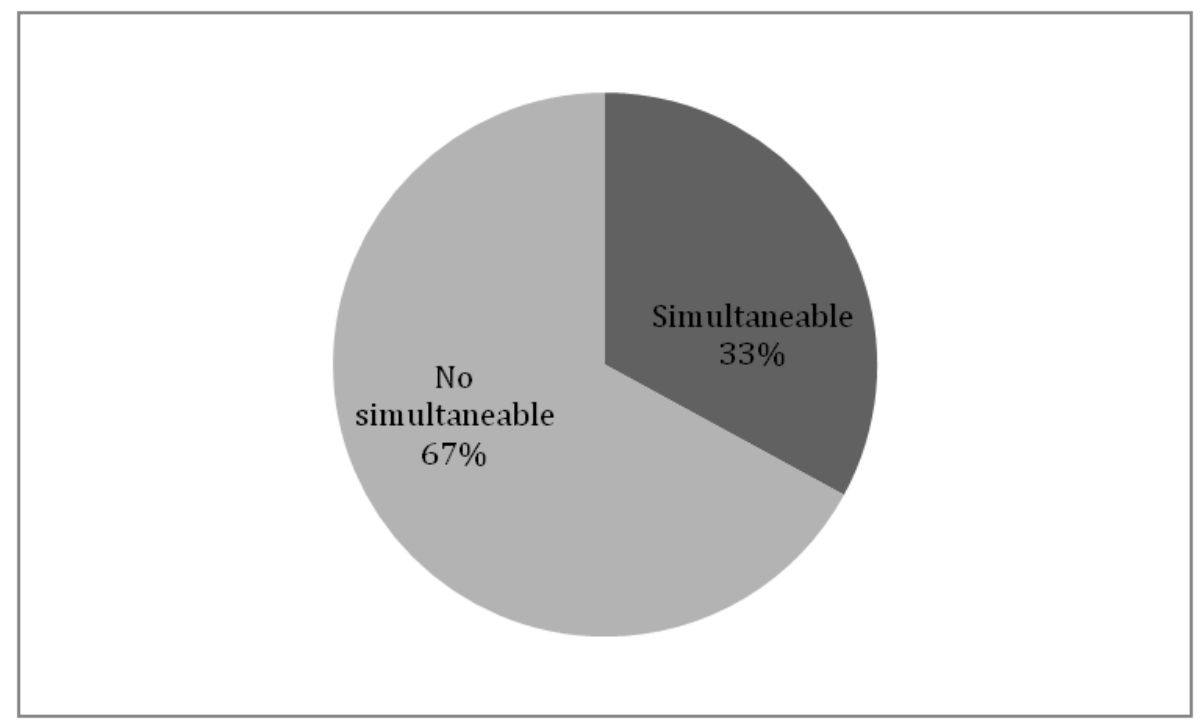

Fuente: elaboración propia

Gráfico 5. Tareas simultaneables según si son físicas o mentales

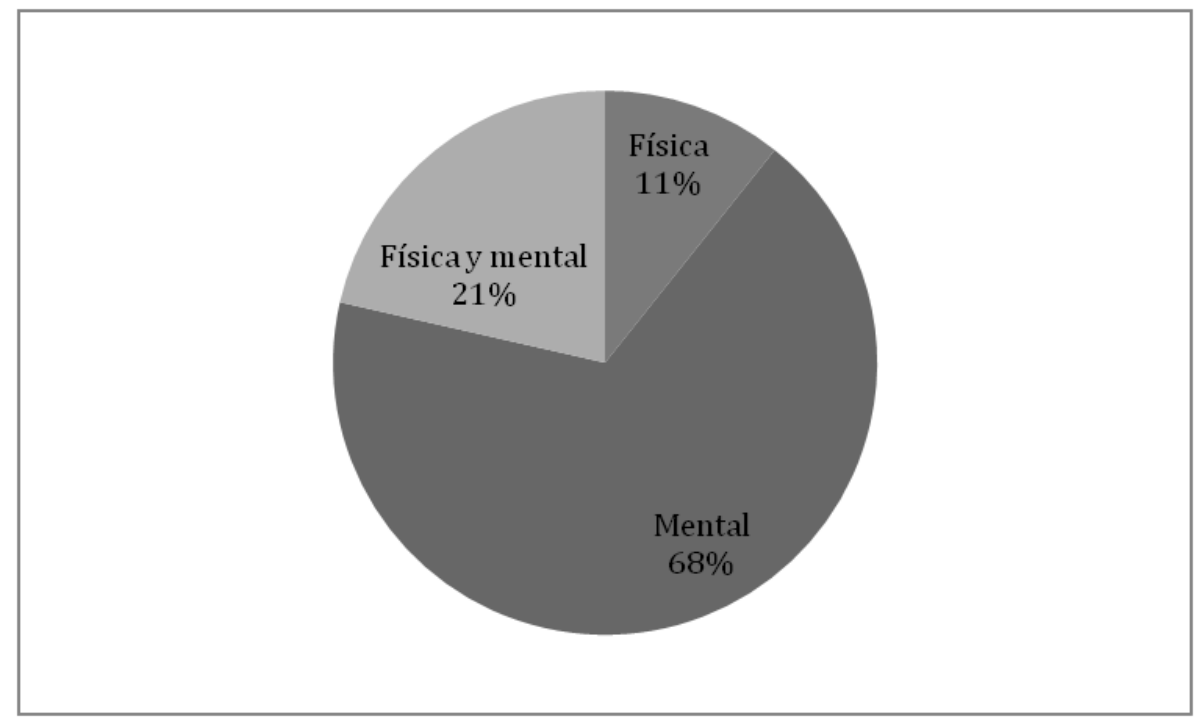

Fuente: elaboración propia 
La siguiente clasificación está relacionada con la flexibilidad de las tareas. Podemos observar (gráfico 6) que casi el $40 \%$ de las tareas tienen la condición de impostergables, lo cual contribuye al estrés que la gestión de las mismas puede suponer sobre aquellas personas que las gestionan, especialmente cuando dichas tareas son asimismo contingentes. En ese sentido destacaremos que el $39 \%$ de las actividades impostergables, según el estudio realizado, resultan contingentes. Ello supone que, en un momento dado, surge una necesidad que no es previsible con anterioridad y a la que hay que hacer frente de forma urgente. El esfuerzo y el estrés generado por estas tareas son, sin duda, muy elevados. Quizás sea quizás este tipo de actividades las que merezcan un estudio pormenorizado en cuanto a sus repercusiones en la persona que es responsable de las mismas, así como lo que ello supone en el desempeño del trabajo y en la armonía de la familia.

\section{Gráfico 6. Impostergable/Flexible}

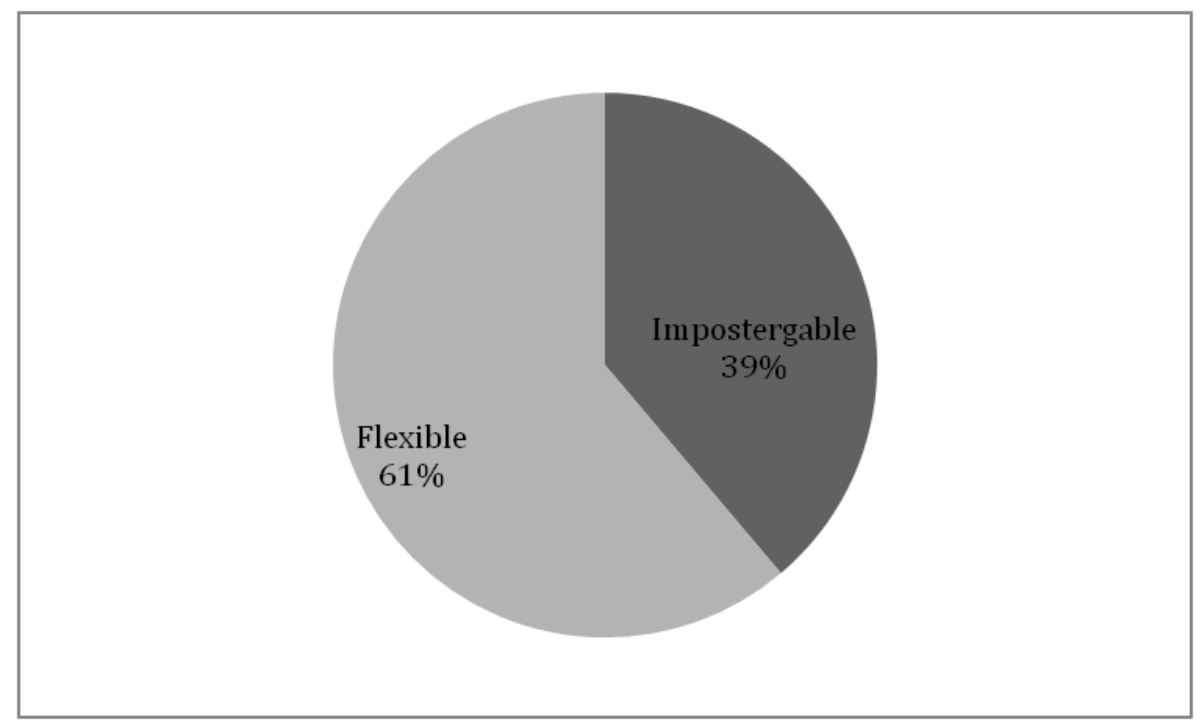

Fuente: Elaboración propia

Respecto a la externalización de las tareas, podemos indicar que un $66 \%$ de las registradas no son externalizables (gráfico 7), y probablemente por ese motivo son menos visibles. La externalización de algunas de estas actividades inicialmente se orienta hacia la familia cercana; cuando no se puede contar con ella o cuando se cuenta con recursos 
económicos suficientes, pueden confiarse a terceras personas que cobran por hacerlo. Lo cierto es que, por un lado, el desarrollo tecnológico, el nuevo concepto de familia y la concepción actual de roles y responsabilidades de sus miembros adultos hace que cada vez haya más actividades que acaban siendo externalizadas; consecuentemente y en relación a ellas, surgen nuevas empresas que se ocupan de ello. Por otro lado, la externalización de una actividad le otorga una mayor visibilización, dado que - especialmente si se tiene que pagar por el servicio prestado - recibe un valor: al ser valorada se vuelve valorable $\mathrm{y}$, consecuentemente, visible. De hecho, hay muchas actividades domésticas y familiares que han conseguido su visibilización gracias, precisamente, a su exteriorización y, por consiguiente, a su asignación de valor económico. Por ese motivo hemos distinguido entre actividades externalizables mercantilizadas, que representarían un $23 \%$ del total de las tareas, y las no mercantilizadas, que representarían solo un $11 \%$ del total de las tareas ${ }^{7}$, como se puede apreciar en el gráfíco 7.

\section{Gráfico 7. Externalizable/No externalizable}

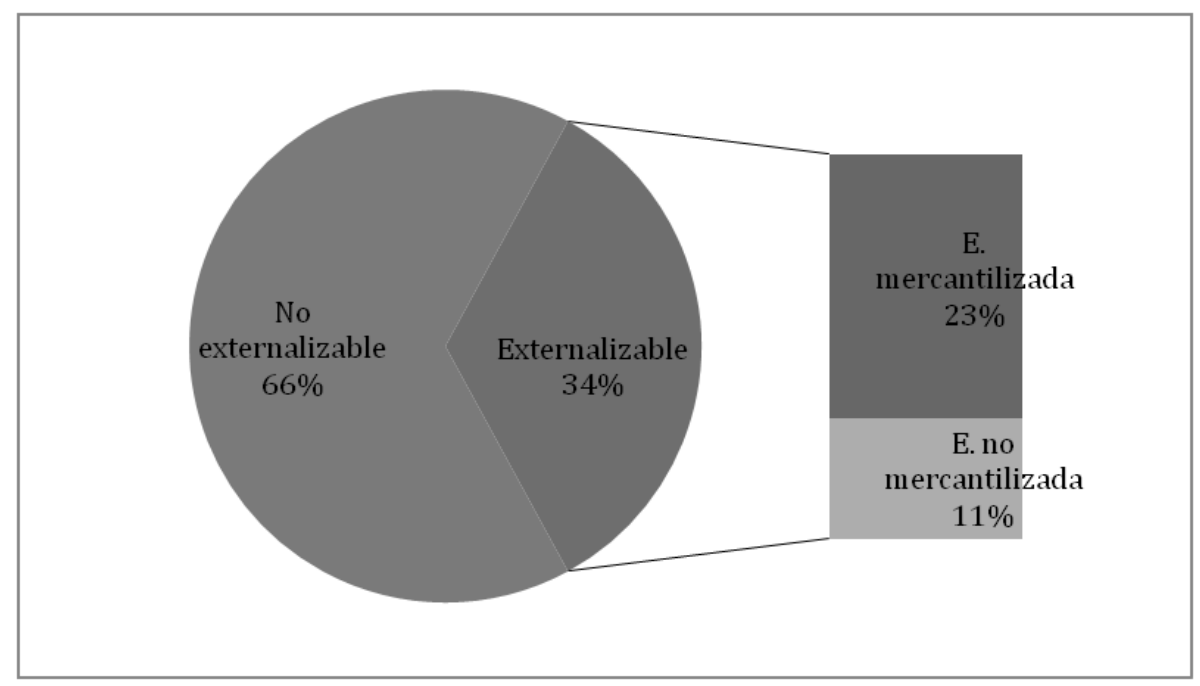

Fuente: elaboración propia

\footnotetext{
${ }^{7}$ Quizás su gratuidad contribuya a su invisibilidad, a que permanezcan ocultas tanto en la agenda de quien las encomienda como de quien las realiza. Precisamente otro foco de estudio podría ser el hacer un análisis por sexos de quienes realizan estas actividades externalizables no mercantilizadas (abuelos y abuelas, parientes cercanos, amistades), de manera que pudiera medirse la sobrecarga que el ayudar a algún familiar o amigo puede suponer para una persona que ya de por sí tiene sus propias tareas y labores cotidianas.
} 
Finalmente, la dicotomía sobre la necesidad o no de desplazamiento separa las actividades con una amplia mayoría — más de tres cuartas partes — hacia las segundas (gráfico 8). Las actividades que no requieren desplazamiento suelen ser las más ocultas en la agenda, sobre todo si se tiene en cuenta que la propia salida del hogar de uno o varios miembros de la familia ya supone, de hecho, una visibilización de la actividad.

\section{Gráfico 8. Con desplazamiento/Sin desplazamiento}

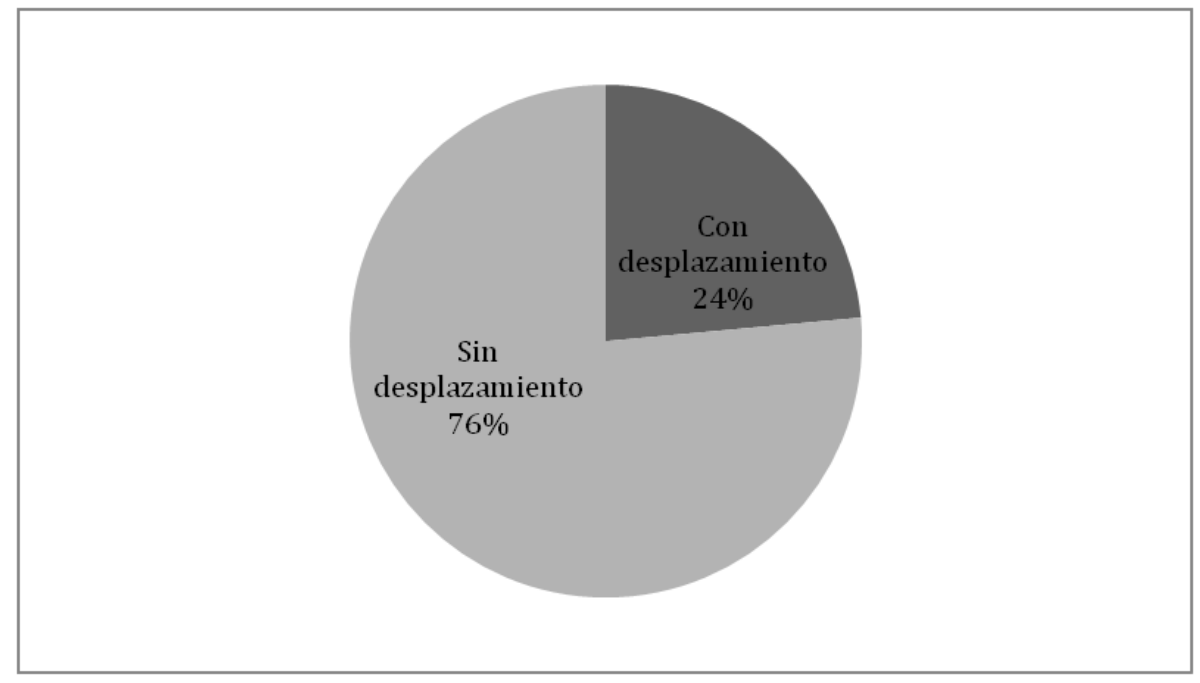

Fuente: elaboración propia

\section{Algunas conclusiones}

Con este trabajo hemos tratado de contribuir a los estudios sobre el trabajo doméstico y los cuidados indagando en aquellas tareas que todavía permanecen invisibilizadas por sus formas residuales y su no monetarización, entre otras razones. Unas actividades en torno a la gestión de la vida y su sostenibilidad, que perfilan la composición de la carga global de trabajo que, mayormente mujeres, asumen en los hogares y respecto a sus familias. La información obtenida en los grupos de discusión ha permitido reflexionar, profundizar y actualizar la noción de trabajo invisible en las rutinas de hombres y mujeres. 
El tipo de actividades que todavía permanecen invisibles son difíciles de operativizar desde una vertiente de uso del tiempo debido a su especificidad, en algunos casos, o a causa de su difusión, en otros. Estas serían las tareas de gestión, organización y cuidados. Con la elaboración de categorías explicativas y la clasificación de las tareas en ellas contenidas, hemos querido elaborar un instrumento cualitativo con el que aprehender aspectos que son más difíciles de evidenciar, como la intensidad y el ritmo con el que estas actividades se llevan a cabo. Es por ello por lo que hemos enfatizado en su cualidad, más que en su cantidad, desplegando categorías significativas que pudieran ofrecer un reflejo más complejo y completo de las implicaciones que supone su asunción.

La participación de hombres en los grupos de discusión ha tenido como pretensión su involucración en la discusión sobre el cuidado de la vida, así como la obtención de información sobre sus percepciones en el reparto y responsabilidad en las tareas que no son de mercado. La disposición de un amplio y diverso abanico de tareas invisibles se convierte así en una herramienta para futuros estudios, tanto cuantitativos como cualitativos, en los que implementar la variable de género de modo que nos ofrezca datos más concretos sobre el reparto de este tipo de tareas.

En definitiva, se trata de un estudio piloto que, confiamos, permitirá abrir nuevas y más amplias vías de exploración en esta dirección. La igualdad —como reclamaba Pérez Royo (2010) hace cinco años—ni está conseguida, ni se producirá espontáneamente, ni puede esperar. Por eso esperamos que nuestro estudio abra líneas de investigación que puedan ayudar a la visibilización de lo invisible en el reparto de la agenda y que, consecuentemente, influyan en la adopción de medidas que lo comprendan y computen. Hay que seguir trabajando para provocar cambios en las políticas que conciernen a la sostenibilidad de la vida, encaminándonos a eliminar la desigualdad en las relaciones de género. La desigualdad, en todas sus formas de expresión, debe seguir siendo un objeto de análisis imprescindible; y la igualdad de oportunidades, un objetivo irrenunciable. 


\section{BIBLIOGRAFÍA}

- Aguirre, Rosario; García Sainz, Cristina y Carrasco, Cristina (2005): El tiempo, los tiempos, una vara de desigualdad. Santiago de Chile: Naciones Unidas, Comisión Económica para América Latina y el Caribe.

- Álvaro Page, Mariano (1996a): "Diferencias, en el uso del tiempo, entre varones y mujeres

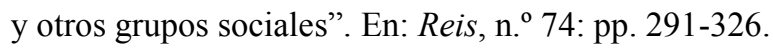

. (1996b): Los usos del tiempo como indicadores de la discriminación entre géneros.

Madrid: Instituto de la Mujer, Ministerio de Asuntos Sociales.

- Ballester Pastor, María Amparo (2012): "La era de la corresponsabilidad: los nuevos retos de la política antidiscriminatoria". En: Lan Harremanak, n. ${ }^{\circ}$ 25, pp. 53-77, [en línea] Disponible en: http://www.ehu.eus/ojs/index.php/Lan_Harremanak/article/view/6172/5820 [12/02/2015].

- Benito Benítez, M. a Angustias (2014): “Feminización de la dependencia: la prestación por cuidados familiares”. En: Cuestiones de género: de la igualdad y la diferencia, n. ${ }^{\circ}$ 9, pp. 338359, [en línea] Disponible en: http://revpubli.unileon.es/ojs/index.php/cuestionesdegenero/article/view/1032 [02/02/2015].

- Cárdenas, Ana, y Undurraga, Rosario (2014): "El sentido del trabajo en mujeres privadas de libertad en Chile”. En: Cuestiones de género: de la igualdad y la diferencia, n. ${ }^{\circ}$ 9, pp. 286309, [en línea] Disponible en: http://revpubli.unileon.es/ojs/index.php/cuestionesdegenero/article/view/1099 [02/02/2015].

- Carrasco Bengoa, Cristina (2013): "El cuidado como eje vertebrador de una nueva economía”. En: Cuadernos de Relaciones Laborales, vol. 31, n. ${ }^{\circ}$ 1, pp. 39-56, [en línea] Disponible en doi: http://dx.doi.org/10.5209/rev_CRLA.2013.v31.n1.41627 [08/02/2015].

- CIRES - Centro de Investigación sobre la Realidad Social (1991): Uso del tiempo. (Encuesta). BBV-BBK-Caja Madrid: Madrid.

. (1993a): Familia y uso del tiempo. (Encuesta). BBV-BBK-Caja Madrid: Madrid.

. (1993b): La vida cotidiana. (Encuesta). BBV-BBK-Caja Madrid: Madrid.

. (1996): Uso del tiempo. (Encuesta). BBV-BBK-Caja Madrid: Madrid.

- Conselleria de Bienestar Social (2007): Decreto 133/2007, de 27 de julio, del Consell, sobre condiciones y requisitos para el visado de los Planes de Igualdad de las Empresas de la 
Comunidad Valenciana, Diari Oficial de la Comunitat Valenciana, n. ${ }^{\circ}$ 5567, de 31 de julio, pp. 31391-31394 [en línea] Disponible en: http://www.uji.es/bin/organs/ui/legisla/auto/d1332007.pdf $[16 / 01 / 2015]$.

- Conselleria de Justicia y Administraciones Públicas (2010): Acuerdo de 9 de abril de 2010, del Consell, por el que se aprueba el Acuerdo de la Mesa Sectorial de Función Pública de 31 de marzo de 2010, por el que se aprobó el I Plan de Igualdad entre Mujeres y Hombres de la Administración de la Generalitat (2010-2012), Diari Oficial de la Comunitat Valenciana, n. ${ }^{\circ}$ 6382, de 22 de octubre, pp. 39275-39352 [en línea] Disponible en: http://www.docv.gva.es/datos/2010/10/22/pdf/2010_11321.pdf [16/01/2015].

- CSIC (Consejo Superior de Investigaciones Científicas) (1994): Encuesta a familias con niños escolarizados. Madrid: CSIC.

. (1995): Encuesta de actividades no remuneradas. Madrid: CSIC.

- Durán, María de los Ángeles (1975): El ama de casa. Crítica política de la economía doméstica. Madrid: Zero-Zyx.

. (1997): "La investigación sobre el uso del tiempo en España: algunas reflexiones metodológicas”. En Revista Internacional de Sociología, n. ${ }^{\circ}$ 18, pp. 163-193.

. (2003): "El trabajo no remunerado en la familia". En Arbor, n. ${ }^{\circ}$ 694, pp. 239-268, [en

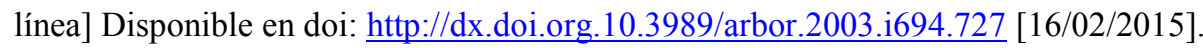

- España (1999): Ley 39/1999, de 5 de noviembre, para promover la conciliación de la vida familiar y laboral de las personas trabajadoras, Boletín Oficial del Estado, n. ${ }^{\circ}$ 266, 6 de noviembre, pp. 38934-38942, [en línea] Disponible en: http://www.boe.es/boe/dias/1999/11/06/pdfs/A38934-38942.pdf [16/01/2015]. . (2007): Ley Orgánica 3/2007, de 22 de marzo, para la igualdad efectiva de mujeres y hombres, Boletín Oficial del Estado, n. ${ }^{\circ}$ 71, 23 de marzo, pp. 12611-12645, [en línea] Disponible en: http://www.boe.es/boe/dias/2007/03/23/pdfs/A12611-12645.pdf [16/01/2015].

- Fernández García, Verónica (2009): “Los trabajos femeninos en el 'Oikos’ de la Grecia Clásica: la madre, la cuidadora, la administradora". En: Cuestiones de género: de la igualdad y la diferencia, . $^{\circ} 4$, pp. 15-50.

- García Sainz, Cristina (2005): “Aspectos conceptuales y metodológicos de las encuestas de uso del tiempo en España”. En: Rosario Aguirre, Cristina García Sainz y Cristina Carrasco: El tiempo, los tiempos, una vara de desigualdad. Santiago de Chile: Naciones Unidas, Comisión Económica para América Latina y el Caribe, pp. 35-50, [en línea] Disponible en: 
https://www.uam.es/personal_pdi/economicas/cgsainz/images/aspectos $\% 20$ conceptuales $\% 20$ ce pal.pdf $[14 / 02 / 2015]$.

- Generalitat Valenciana (2003): Ley 9/2003, de 2 de abril, de la Generalitat, para la Igualdad entre Mujeres y Hombres, Diari Oficial de la Comunitat Valenciana, n. ${ }^{\circ} 4474$, de 4 de abril, [en línea] Disponible en: http://www.docv.gva.es/portal/ficha_disposicion.jsp?id=24\&sig=1587/2003\&L=1\&url_lista $=$ [16/01/2015].

. (2013): I Plan de Igualdad entre Mujeres y Hombres de la administración de la Generalitat 2010-2012, [en línea] Disponible en: http://web.ua.es/es/unidadigualdad/documentos/planes-de-igualdad/plan-igualdad-administracion-generalitat.pdf [16/01/2015].

- Hurtado, Inmaculada (2010): “Now it's my turn. Inmigración de retiro y cuidados”. En: Esteban, Mari Luz, Comelles, Josep Maria, y Díez Mintegui, Carmen (eds.): Antropología, género, salud y atención. Barcelona: Bellaterra.

- Krueger, Richard A. (1997): Moderating Focus Groups (Focus Group Kit). Thousand Oaks: Sage Publications.

- Marcos Santiago, María del Rosario (2005): "Reflexiones acerca del trabajo de las mujeres". En: Cuestiones de género: de la igualdad y la diferencia, n. ${ }^{\circ}$ 0, pp. 111-153.

. (2009): "El largo recorrido hacia el reconocimiento social y económico del trabajo de las mujeres”. En: Cuestiones de género: de la igualdad y la diferencia, n. . 4, pp. 1-14.

- Martín Llaguno, Marta; León Llorente, Consuelo y Guirao Mirón, Cristina (2013): “La conciliación familiar y laboral en España en la esfera política, mediática y empresarial. Un estudio de agendas entre 2003 y 2007”. En: Cuestiones de género: de la igualdad y la diferencia, n. ${ }^{0} \quad 8, \quad$ pp. 145-164, [en línea] Disponible en: http://revpubli.unileon.es/ojs/index.php/cuestionesdegenero/article/view/883 [15/01/2015].

- Merton, Robert King, Fiske, Majorie, y Kendall, Patricia L. (1956): The Focused Interview. A Manual of Problems and Procedures. New York: Fee Press.

- Morgan, David L. (1988): Focus Group as Qualitative Research. Newbury Park: Sage.

- Núñez, Trinidad, y Loscertales, Felicidad (2003): El grupo y su eficacia. Técnicas al servicio de la dirección y coordinación de grupos (3. ${ }^{\mathrm{a}}$ ed.). Barcelona: Octaedro.

- Pérez Royo, Javier (2010): “La igualdad no puede esperar". En: El País, 16 de abril, n. 11.985 , p. 23. 
- Rodríguez, Arantxa y Larrañaga, Mertxe (2003): El trabajo de las mujeres: claves para entender la desigualdad laboral. Vitoria: EMAKUNDE, Instituto Vasco de la Mujer.

- Royo, Raquel (2003): "Trabajo doméstico desde la perspectiva de género. Estudio cualitativo". En: María Luisa Setién y María Silvestre (eds.): Problemas de las mujeres, problemas de la sociedad. Bilbao: Universidad de Deusto, pp. 181-192.

- Sánchez Reinón, Manuel (2012): “Los ‘cuidados informales’ de larga duración en el marco de la construcción ideológica, societal y de género de los 'servicios sociales de cuidados'. En: Cuadernos de Relaciones Laborales, vol. 30, n. ${ }^{\circ}$ 1, pp. 185-210, [en línea] Disponible en doi: http://dx.doi.org.10.5209/rev_CRLA.2012.v30.n1.39120 [08/02/2015].

- Saneleuterio, Elia (2010): “Espacio público y territorialización. La imagen de la mujer en la obra de José Hierro”. En: María Mercedes González de Sande (cur.): Donne, identità e progresso nelle culture mediterranee. Roma: Aracne, pp. 429-440.

- Setién Santamaría, María Luisa (2003): “Los tiempos de las mujeres. Una manifestación de la desigualdad”. En: María Luisa Setién y María Silvestre (eds.): Problemas de las mujeres, problemas de la sociedad. Bilbao: Universidad de Deusto, pp. 63-84.

- Sierra, Restituto (2003): Técnicas de investigación social. Teoría y ejercicios (14. ${ }^{\mathrm{a}}$ ed.). Madrid: Paraninfo. 